\title{
Re-evaluation of the Indication and Limitation of Laparoscopic Salpingotomy for Tubal Pregnancy
}

\author{
Akira Fujishita $^{1 *}$, Khaleque Newaz $\operatorname{Khan}^{1}$, Michio Kitajima ${ }^{1}$, Koichi Hiraki ${ }^{1}$, \\ Seiyou Miura $^{1}$, Tadayuki Ishimaru ${ }^{2}$, Hideaki Masuzaki ${ }^{1}$ \\ ${ }^{1}$ Department of Obstetrics and Gynecology, Nagasaki University School of \\ Medicine, Nagasaki, ${ }^{2}$ Sasebo Chuo Hospital, Nagasaki, Japan \\ * Current affiliation: Department of Obstetrics and Gynecology, Nagasaki \\ Municipal Hospital, Nagasaki, Japan
}

Running Title: Indication of successful laparoscopic salpingotomy

Address for Correspondence and Reprint Requests:

Akira Fujishita, M.D

Department of Obstetrics and Gynecology

Nagasaki Municipal Hospital

6-39 Shinchi, Nagasaki 850-8555, Japan.

Tel: $+81-95-8223251$

Fax: +81-95-8269798

E-mail: sanka_fujishita@nmh.jp 
Fujishita A et al. Page 2

\section{Condendation}

The increased size and proximal anatomic location of the gestational

sac in the fallopian tube do not impair the success rate of laparoscopic salpingotomy and can be equally practiced as a conservative surgery. 
Title of article: Re-evaluation of the Indication and Limitation of Laparoscopic Salpingotomy for Tubal Pregnancy

Name of authors: A Fujishita, KN Khan, M Kitajima, K Hiraki, S Miura, T Ishimaru, H Masuzaki

\begin{abstract}
Objective. We investigated the outcome of laparoscopic salpingotomy for tubal pregnancy by follow-up hysterosalpingography (HSG) or second look laparoscopy (SLL) and reexamined the indication and limitation of this conservative surgery.
\end{abstract}

Study Design. From April 1991 to December 2003, we treated one hundred and eighty one cases of tubal pregnancy by laparoscopic salpingotomy. The tubal patency was assessed by either HSG or SLL performed at three months post-surgery. The successful patients at initial operation and the confirmed ipsilateral patent tubes at follow-up were classified as true successful cases (Group I). Even after successful operation, if the treated tubes were found occluded, they were considered as failure cases. Therefore, unsuccessful cases at initial surgery as well as at follow-up were categorized as Group II. 
Results. One hundred thirty four cases (74\%) were successfully treated by salpingotomy at initial laparoscopy and also 85 of them (63.4\%) were found truly successful at follow-up (Group I). The remaining 47 cases (26.0\%) were unsuccessful at initial surgery and 18 (13.4\%) cases at follow-up (Group II). Other patients who refused to accept a tubal patency test or not examined as a result of personal reason or lost follow-up comprised 31 cases. No difference in surgical outcome was observed with gestational age, intra-operative hemorrhage, size or anatomic location of pregnancy mass, and preoperative adhesions of the fallopian tube between these two groups of patients. However, pre-operative serum levels of hCG were significantly higher in Group II compared with Group I. In addition, the failure cases were more frequently associated with positive fetal heart beat (FHB), tubal rupture and a preoperative serum levels of hCG of more than $10,000 \mathrm{IU} / \mathrm{L}(\mathrm{p}<0.05, \chi 2$ test $)$. The Log-rank test indicated a higher success in achieving pregnancy in Group $\mathrm{I}(\mathrm{p}<0.05)$ than in Group II who desired for future pregnancy.

Conclusion. Laparoscopic salpingotomy can be equally practiced as a conservative surgery for also proximal ectopic pregnancy and gestational 
mass size is not so much important and not a relative contraindication for conservative laparoscopic surgery as previously reported. Low preoperative hCG levels, absence of FHB, absence of tubal rupture initially or minimal rupture may be considered as suitable parameters for successful surgery and achieving future pregnancy.

Key Words: ectopic pregnancy, laparoscopy, linear salpingotomy, pregnancy outcome 


\section{Introduction}

The diagnosis and management of ectopic pregnancy have changed dramatically with the technical advances in sensitive assays for human chorionic gonadotropin (hCG) and widespread use of transvaginal sonography (TVS). As a result, ectopic pregnancy can be identified early and medical or surgical therapy can be given promptly. Evidences support a conservative approach to the tubal pregnancy in women wishing to preserve their future fertility [1-4].

Intrauterine pregnancy rates and the incidence of recurrent ectopic pregnancy in these studies appear as good as or better than the results following salpingectomy. This conservative surgery initially involved laparotomy, linear salpingotomy, and removal of intra-luminal blood clots and products of conception. Recent modifications in the laparoscopic instrument and technique have permitted more satisfactory endoscopic approach to ectopic pregnancy, with a potential reduction in morbidity and duration of hospitalization [5-8].

Previous studies have demonstrated the indication of conservative surgery for tubal pregnancy by laparoscopy as almost the same considerable 
criteria as laparotomy $[2,5,9,10]$. Tubal size, preoperative levels of hCG, detection of fetal heart beat (FHB) by TVS, and tubal rupture was among the important criteria to predict management outcome [11-13]. However, information regarding the true successful rate is limited in previous studies. This true successful case was defined when the initial surgery was success and also the treated (ipsilateral) tube was patent on HSG or SLL. Therefore we attempted to re-evaluate the true successful or failure cases after initial surgery and reexamined the indication and limitation of laparoscopic salpingotomy for tubal pregnancy in clinical practice. We also prospectively evaluated the difference in future pregnancy rate between successfully operated cases and unsuccessful cases.

\section{Materials and Methods}

2.1. Subjects. Between April 1991 and December 2003 a total 394 patients were operated on for ectopic pregnancy (EP) at the Department of Nagasaki University Hospital. We performed laparoscopic salpingotomy in the studied women with the following criteria: (1) the patients' who desire for future pregnancy, (2) hemodynamically stable women as defined by normal arterial 
pressure and heart rate and whose hematocrit follow-up showed no decreasing, (3) no signs of active bleeding as defined by lack of either arterial or venous bleeding from the ruptured site at laparoscopy, and (4) occurrence of filmy adhesions in tubal wall. All interstitial, ovarian and abdominal pregnancies were excluded from this study. Finally 181 tubal pregnancies were treated by laparoscopic salpingotomy during this period. This study was approved by the local institutional review board (IBR) of Nagasaki University. A preoperative informed consent was obtained from all women after explaining them the possible risks and complications of conservative procedures such as persistent ectopic pregnancy and repeated tubal pregnancy. Linear salpingotomy was the most feasible choice for those patients desiring future fertility. If tubal damage precluded a linear salpingotomy, a radical management such as segmental resection or salpingectomy was performed as appropriate.

2.2. Surgical procedures. After confirmation of the tubal pregnancy by diagnostic laparoscopy, a modified operative technique was performed in all patients as previously described $[11,14,15]$. In the case of any persisting 
bleeding or oozing of blood from the fallopian tube as occurred in possible tubal damage, the salpingotomy procedure was switched to the radical treatment.

2.3. hCG assays and follow-up. The serum levels of human chorionic gonadotropin (hCG) were measured by IMMULYZE (Diagnostic Product Corporation, USA) assay. The limit of detection was $1.0 \mathrm{IU} / \mathrm{L}$ and both the intra-assay and inter-assay coefficients of variation were $<7.0 \%$ for these assays.

The cases with persistent ectopic pregnancy were identified by serial monitoring of serum hCG levels during post-operative period and were defined by the re-elevation or failure to decline of hCG levels after initial surgery. In such a case, intra-muscular methotrexate (MTX) was injected as described previously [15]. The patients who complained of persistent abdominal pain and showed subsequent further increase in hCG levels after MTX injection were considered as failure cases and a second look laparoscopy (SLL) was allocated for these cases.

Patients were instructed to maintain contraception for three months 
after the first operation. The tubal patency was assessed by either HSG or SLL performed at three months post-surgery. We recommended at first the SLL as the most precise test to assess tubal condition. The follow-up period ranged from one month to 88 months (mean 20.2 months). Patients were inquired if they were attempting pregnancy and if so, for how long. Pregnancy outcome was evaluated time-dependently in a group of these patients.

2.4. Statistical analysis. Data were analyzed by using either a Student's t-test or Wilcoxon's rank sum test or $\chi^{2}$ test. In the event of pregnancy, the cumulative pregnancy rate was calculated by the inverse curve analysis of the Kaplan-Meier methods and the difference between curves was evaluated by Log-rank test. A value of $\mathrm{p}<0.05$ was considered as significant. All data are expressed as means $\pm \mathrm{SD}$.

\section{Results}

One hundred thirty four cases (74.0\%) were successfully treated by salpingotomy at initial laparoscopy. However forty-seven patients (26.0\%) 
were unsuccessful at initial surgery because of heavy bleeding or complete tubal damage and these 47 cases were switched to either segmental resection or salpingectomy. The initial EP was located in the right tube of one hundred three cases and in the left tube of seventy-eight cases.

Among one hundred thirty four cases of successful linear salpingotomy, ipsilateral tube was found patent in eighty-five women (63.4\%) after three months of follow-up by HSG $(n=48)$ or SLL $(n=37)$ and was considered as a group of true successful cases (group I). In eighteen of 134 successful cases at initial surgery, the treated tubes were found occluded by follow-up HSG $(n=15)$ or SLL $(n=3)$. Therefore, forty-seven unsuccessful cases at initial surgery together with 18 unsuccessful cases at follow-up constituted the total 65 (35.9\%) unsuccessful cases (group II). The remaining thirty one of 134 successful cases at initial surgery did not satisfy the inclusion criteria for following reasons: five patients were conceived within three months even kept on contraception for at least three months, nineteen patients were refused to accept HSG because they were single or divorced thereafter, and other seven cases were lost in the follow-up period (Figure 1). 
The clinical and intra-operative demographic data of women in group I and group II are shown in Table 1. There was no significant difference in any clinical profiles between these two groups of women except serum hCG levels. The pre-operative serum levels of hCG were significantly higher in unsuccessful cases (group II) compared with those of successful cases (group I) $(14,583 \pm 27,498$ versus 2,103 $\pm 2,185$ IU/L, respectively; p<0.0001). (Table 1 )

To predict more accurate indication of true successful cases at linear salpingotomy, we examined various anatomical and diagnostic parameters as observed during surgery or before surgery (Table 2). There was no significant difference in any of the parameters between group I and group II women except in tubal condition, pre-operative hCG levels and detection of FHB by TV-USG (Table 2).

The rupture of the tube was seen in total twelve cases, three cases (25\%) displayed tubal patency but nine cases (75\%) showed occluded ipsilateral tubes by follow-up HSG or SLL $\left(\mathrm{p}<0.05\right.$ for both by $\chi^{2}$ test). In this series of our study, there were no cases showing a maximum size of more than $8.0 \mathrm{~cm}$ of EP lesion. The success rate of the surgery was $65 \%$ 
$(15 / 23$ cases $)$ for the lesion with a maximum size of less than $2.0 \mathrm{~cm}$, and $58 \%(49 / 85$ cases $)$ from $2.0 \mathrm{~cm}$ to $5.0 \mathrm{~cm}$, and $50 \%(21 / 42$ cases $)$ for a size of more than $5.0 \mathrm{~cm}$ in diameter. There were no statistical differences in the success rate of EP among these three sizes. In fact, success rate was almost similar for $<2 \mathrm{~cm}, 2-5 \mathrm{~cm}$ and $>5 \mathrm{~cm}$ sizes of gestational masses, which indicates that the size of the EP did not affect the success rate.

When the serum levels of hCG were higher than 10,000 IU/L, the unsuccessful rate of the surgery was $100 \%(25 / 25$ cases $)\left(p<0.001, \chi^{2}\right.$ test $)$ and surgical successes were obtained for all cases with serum hCG levels of less than 10,000 IU/L (Table 2). The lower the preoperative serum hCG levels, the more success in surgery was observed and vice versa.

The presence of fetal heart beat (FHB) in the gestational sac was confirmed in total sixteen cases by preoperative TVS. Among them, only two cases (case No.1 and No.6) (Table 3) belonged to successful group but other fourteen cases belonged to unsuccessful group. A close relationship was observed between success in surgery and absence of pre-operative detection of FHB by TV-USG $(\mathrm{p}<0.001)$ (Table 3$)$. The detail clinical profiles and consequence of cases with FHB are described in Table 3. 
In the evaluation of pregnancy outcome, single women, women using contraception or divorced women were excluded. Therefore, 70 women of 85 cases in group I and 52 women of 65 cases in group II were enrolled who were actively trying to naturally conceive. Among them, 53 (75.7\%) cases in group I and $23(44.2 \%)$ cases in group II were conceived during the follow-up period (1-88 months, mean 20.2 months). There was no statistical difference between the groups. The distribution of the pattern of pregnancy outcome between these two groups is shown in Table 4.

When we analyzed the cumulative pregnancy rate by Log-rank test, pregnancy rate was found to be significantly higher in Group I $(52.5 \%$ at 6 months, $73.5 \%$ at 12 months, $78.3 \%$ at 18 months, $81.4 \%$ at 24 months and $86.0 \%$ at 36 months) than that observed in Group II (31.7\% at 6 months, $42.8 \%$ at 12 months, $52.5 \%$ at 18 months, $56.9 \%$ at 24 months and $56.9 \%$ at 36 months $)(p=0.0036$, Log-rank test, Figure 2).

\section{Discussion}

In this study we strictly evaluated the success rate and failure rate of patients with tubal pregnancy treated with laparoscopic salpingotomy by 
their on-going outcome at initial surgery and their subsequent tubal condition at follow-up. Combining these two different observations at initial surgery and at follow-up, we found a success rate of 56.7\% (85/150) for true successful cases and $43.3 \%(65 / 150)$ for unsuccessful cases. This surgical outcome in our study was irrespective of the anatomic location of the EP lesion on the fallopian tube and their size. More than half of these patients achieved an intrauterine pregnancy and only $13.2 \%$ (7/53) of women displayed a repeated tubal pregnancy.

A few published reports were available until now on tubal pregnancy involving isthmic part. Recently Chapron et al. [10] reported the conservative laparoscopic treatment of isthmic ectopic pregnancy. Their therapeutic approach was very reliable since only one failure case was observed (3.9\%). It was possible to evaluate subsequent fertility for 11 cases (63.6\%) achieving intrauterine pregnancy and only one patient $(9.1 \%)$ had a recurrence. In our series, the success rate of surgery for isthmic pregnancy was $38 \%$ and these cases retained tubal patency by follow-up HSG or SLL. We assume that the isthmic pregnancy should not always be contraindicated for laparoscopic conservative surgery if careful management is offered. 
Rupture of ectopic pregnancy has been considered as a contraindication to the laparoscopic conservative surgery $[2,10]$, although laparoscopic salpingectomy has been accomplished successfully in selected cases [12]. In our study only three ruptured cases (25\%) were successful in laparoscopic conservative surgery. Among these three successful cases, one patient was suffered by a repeat ipsilateral tubal pregnancy, and one case achieved an intrauterine pregnancy. Thus we postulate that conservative surgery can be adapted in some selected cases in which the size of the rupture is small, when there is no active bleeding, in patients with relatively stable condition, and for women who strongly wish for future pregnancy after obtaining informed consent.

In this study we can add new information that tubal size is not related to the successful outcome of laparoscopic salpingotomy for tubal pregnancy. Our findings are not in consistent with that reported recently by others. DeCherney et al. [10] considered ectopic pregnancy having a diameter of $3 \mathrm{~cm}$ as a reasonable upper limit for the success of linear salpingotomy. Pouly et al. [11] indicated absolute limit to $6 \mathrm{~cm}$ and regarded a diameter of more than $4 \mathrm{~cm}$ as a relative contraindication. Vermesh [2] 
recommended that the laparoscopic approach probably should not be attempted when the maximum diameter of EP lesion is more than $5 \mathrm{~cm}$. However, the true success rate for the EP lesion of more than $5 \mathrm{~cm}$ in the ampullary part was $50 \%$ in our current study. Indeed, diameter of the EP lesion in some cases was over $5 \mathrm{~cm}$ and in such cases intra-tubal contents were filled with blood and clots. With careful approach, it was possible to remove all residual contents of the involved tube resulting in consequent shrinkage of respective swollen tube. All ectopic pregnancies involving the ampullary part in our series carried a longitudinal diameter of more than $5 \mathrm{~cm}$ and none of these cases involved the isthmic part. The maximum diameter of isthmic part in our successful cases was $3 \mathrm{~cm}$ in length. Again, size of EP in the ampullary area was also variable. Five centimeter in thirteen cases and $6 \mathrm{~cm}$ in six cases and a maximum of $7 \mathrm{~cm}$ was observed in two cases. However, no successful surgery was observed for a lesion of more than $8 \mathrm{~cm}$ in diameter. Combining the different sizes, our true successful rate was relatively high and no side effect was observed after initial operation. Thus we recommend that if the size of ectopic pregnancy is about $5-6 \mathrm{~cm}$ and present with suitable biophysical compliance of the patients, it can be 
possible to perform linear salpingotomy for these cases by laparoscopy.

In our results, pre-operative serum hCG levels were significantly higher in failure cases compared with successful cases. Our results are in agreement with some reports from other laboratory. Pouly et al. [11] reported that preoperative hCG levels of more than 15,000 IU/L was an absolute contraindication for surgery. Milad et al. [16] described that a preoperative serum beta-hCG levels of more than 8,000 IU/L was highly predictive for the failure of linear salpingotomy by laparoscopy. A further evaluation by Colacurci et al. [17] demonstrated that preoperative hCG values of either less or more than 10,000 IU/L adversely affected the operative course, tubal patency and subsequent reproductive performance. In fact, no true successful cases were observed with a pre-operative serum hCG levels of more than 10,000 IU/L in our current study.

Most of the FHB-positive cases were also associated with higher pre-operative hCG levels of more than $10,000 \mathrm{IU} / \mathrm{L}$. In the case of positive FHB, risk of bleeding increased from the surface or deeper site of incision due to hyper-vascular EP lesion causing very difficult removal of the conceptus. In our study, surgery was successful in only two FHB-positive 
cases but in clinical practice these cases were rare and were found by chance. Therefore, when a tubal pregnancy is associated with a positive FHB or with a pre-operative hCG levels of more than 10,000 IU/L, we must contrive way to less bleeding or should find the suitable approach for successful reproductive outcome. Management of these high risk cases by a combination of laparoscopic salpingotomy with local injection of methotrexate can be an approach or laparoscopic tubal anastomosis after segmental resection, a microsurgical procedure [18, 19], can be reserved for any future demand.

Persistent EP was a common complication and the major reason for secondary intervention after laparoscopic salpingotomy. In this study, no statistical difference was observed in the development of persistent EP between the groups. A recent study claimed that neither clinical variables nor single measurement of serum hCG could be a predictor for accurate diagnosis of persistent EP [20].

As shown in Table 2, it is obvious that increased tubal rupture, a higher serum hCG levels of more than 10,000 IU/L and TVS-detection of fetal heart beat were responsible for the unsuccessful event at intial surgery. 
It may be speculated that preoperative hCG levels higher than 10,000 IU/L means almost bad prognosis but hCG levels lower than 10,000 IU/L does not always assure good prognosis, as seen in our cases. It is also clear from Table 2 that increased un-ruptured condition of the tube (82 cases of 85 cases), a lower serum hCG levels of less than 10,000 IU/L (85 of 85 cases) and less findings of fetal heart beat by TVS were responsible for the successful outcome of initial surgery. These can be accepted as suitable parameters to improve the successful rate.

In conclusion, we suggest that location of ectopic mass and gestational mass size is not so much important and should not be accepted as a relative contraindication for conservative laparoscopic surgery as previously reported. Low preoperative HCG levels, absence of FHB, and absence of tubal rupture initially or minimal rupture may be considered as suitable parameters for successful surgery and achieving future pregnancy. We believe that our indications and limitations in the laparoscopic conservative surgery can be useful in clinical practice to carefully manage the on-going surgery and to improve future pregnancy. 


\section{References}

[1] Lavy G, Diamond MP, DeCherney AH: Ectopic pregnancy: its relationship to tubal reconstructive surgery. Fertil Steril 1987; 47: 543-556.

[2] Vermesh M: Conservative management of ectopic gestation. Fertil Steril 1989; 51: 559-567.

[3] Korell M, Albrich W, Hepp H: Fertility after organ-preserving surgery for ectopic pregnancy: results of multicenter study. Fertil Steril 1997; 68: $220-223$.

[4] Mol BWJ, Matthijsse HC, Tinga DJ, et al: Fertility after conservative and radical surgery for tubal pregnancy. Hum Reprod 1998; 13: 1804-1809.

[5] DeCherney AH, Diamond MP: Laparoscopic salpingostomy for ectopic pregnancy. Obstet Gynecol 1987; 70: 948-950.

[6] Maymon R, Shulman A, Halperin R, et al: Ectopic pregnancy and laparoscopy: review of 1197 patients treated by salpingectomy or salpingostomy. Eur J of Obstet Gynecol 1995; 62: 61-67.

[7] Yao M, Tulandi T: Current status of surgical and nonsurgical management of ectopic pregnancy. Fertil Steril 1997; 67: 421-433.

[8] Hajenius PJ, Mol BWJ, Bossuyt PMM, et al: Interventions for tubal 
ectopic pregnancy. Cochrane Database Syst Rev 2000; 2: CD000324.

[9] DeCherney AH, Boyers SP: Isthmic ectopic pregnancy: segmental resection as the treatment. Fertil Steril 1985; 44: 307-312.

[10] Chapron C, Pouly JL, Wattiez A, et al: Results of conservative laparoscopic treatment of isthmic ectopic pregnancies: a 26 case study. Hum Reprod 1992; 7: 422-424.

[11] Pouly JL, Mahnes H, Mage G, et al: Conservative laparoscopic treatment of 321 ectopic pregnancies. Fertil Steril 1986; 46: 1093-1097.

[12] DeCherney AH, Romero R, Naftolin F: Surgical management of unruptured ectopic pregnancy. Fertil Steril 1982; 35: 21-24.

[13] Reich H, Freifeld ML, McGlynn F, et al: Laparoscopic reatment of tubal pregnancy. Obstet. Gynecol 1987; 69: 275-279.

[14] Tulandi T, Saleh A: Surgical management of ectopic pregnancy. Clin Obstet Gynecol 1999; 42: 31-38.

[15] Fujishita A, Masuzaki H, Khan KN, Kitajima M, Hiraki K, Ishimaru T. Laparoscopic salpingotomy for tubal pregnancy: comparison of linear salpingotomy with and without suturing. Human Reprod 2004; 19: 1195-1200. 
[16] Milad MP, Klein E, Kazer RR: Preoperative serum hCG level and intraoperative failure of laparoscopic linear salpingostomy for ectopic pregnancy. Obstet Gynecol 1998; 92: 373-376.

[17] Colacurci N, Zarcone R, Franciscis PD, et al: Tubal patency after laparoscopic treatment of ectopic pregnancy. Panminerva Med 1998; 40: 45-47.

[18] Templeman C, Davis C, Janik G, et al: Laparoscopic microsurgical anastomosis of the blocked, solitary post-ectopic fallopian tube. Human Reprod 2002; 17: 1630-1632.

[19] Jeffrey M, Goldberg M, Falcone T: Laparoscopic microsurgical tubal anastomosis with and without robotic assistance. Human Reprod 2003; 18: $145-147$.

[20] Lund CO, Nilas L, Bangsgaard N, et al: Persistent ectopic pregnancy after linear salpingostomy: a non-predictable complication to conservative surgery for tubal gestation. Acta Obstet Gynecol scand 2002;81:1053-1059. 


\section{Figure Legends}

Figure 1. Shows the flow chart of the cases in the successful group and unsuccessful group. After final exclusion of 18 cases at follow-up and 31 cases who did not the meet the inclusion criteria, a total of 85 cases were enrolled in group I as successfully operated cases and a total of 65 cases in group II as unsuccessful cases. The details in the selection of these two groups of patients are described in materials and methods.

Figure 2. Shows the cumulative pregnancy rate in the successfully operated cases (Group I) and in unsuccessful cases (Group II) during the post-operative follow-up period. The inverse curve analysis of pregnancy outcome was calculated by Kaplan-Meier methods and the Log-rank test indicated a significantly higher pregnancy rate in group I than that in group II $(\mathrm{p}=0.0036)$. 